\title{
ASYMPTOTICS AND RANDOM MATRICES WITH ROW-SUM AND COLUMN SUM-RESTRICTIONS ${ }^{1}$
}

\author{
BY PATRICK EUGENE O'NEIL
}

\author{
Communicated by Gian-Carlo Rota, July 3, 1969
}

1. Introduction. We desire to use the inclusion-exclusion formula for determining asymptotic approximations. This object was first achieved for a special problem by P. Erdös and I. Kaplansky [1]. The following indicates the form of the asymptotic estimate we may obtain.

Let $S$ be an arbitrary finite set, each element of which may be said to possess "properties" from an $L$-set $P$ of properties $\left\{P_{1}, P_{2}, \cdots, P_{L}\right\}$. Let

$$
N\left(P_{i_{1}}, P_{i_{2}}, \cdots, P_{i_{j}}\right)
$$

be the number of elements in the set $S$ which possess all the properties of the set $\left\{P_{i_{1}}, P_{i_{2}}, \cdots, P_{i_{j}}\right\}$, and possibly more. To define $s_{j}$, let

$$
N(0) s_{j}(j !)^{-1}=\sum N\left(P_{i_{1}}, P_{i_{2}}, \cdots, P_{i_{j}}\right),
$$

where the sum runs over all $j$-subsets of $P$. Let us formally define $\left(s_{1}\right)^{j}=s_{j}$, with $s_{j}=0$ for $j>L$. Then if $E(0)$ denotes the number of elements of $S$ with none of the properties of $P$, we may formally represent $E(0)$ by

$$
E(0)=N(0) e^{-s_{1}}
$$

This representation is merely the sieve formula or the simple inclusion-exclusion formula in a formal guise. It turns out that for a great many problems of interest, this formal equation is a valid asymptotic approximation when certain restrictions are placed on the properties of $P$.

Some notation is required for the results which follow. Let $M^{n}(R, S)$ be the class of $n \times n(0-1)$-matrices with row-sum vector $R$ and column-sum vector $S$. We denote by $r_{i}$ or $s_{i}$ the $i$ th component of the $n$-length vector $R$ or $S$ respectively. It is always assumed that $\sum r_{i}$ $=\sum s_{i}$. We further restrict the vectors $R$ and $S$ so that the number $N$ of integers $i$ in $\{1,2, \cdots, n\}$ such that $r_{i}=0$ or $s_{i}=0$ is very small for large $n: N=O(\log n)$. The symbol $M^{n}(k, k)$ designates the class

1 This work is a major portion of the author's dissertation for $\mathrm{a} \mathrm{PhD}$, received at the Rockefeller University. 
of $n \times n(0-1)$-matrices all of whose row sums and column sums are $k$. E.g.: $M^{n}(1,1)$ is the class of permutation matrices.

We shall hereafter use the tablemaker's notation, $i=1(1) n$, to signify that $i$ ranges from 1 to $n$ by steps of 1 .

Given an $n \times n$ matrix $F$, called a "frame," a matrix $A \in M^{n}(R, S)$ is said to "lie in" the frame $F$ iff $F(i, j)=1$ whenever $A(i, j)=1$, $i=1(1) n, j=1(1) n$.

$M^{n}(R, S)$ is said to be a class of sparse matrices with associated function $f(n)=o(n)$ if $r_{i}<f(n)$ and $s_{i}<f(n), i=1(1) n$. Depending on the function $f(n)$, the sparseness of the matrices in $M^{n}(R, S)$ may be a very weak restriction for a particular $n$; we may consider this property to be meaningful only as $n$ ranges: given $f(n)$, then as $n$ approaches infinity all the matrices which are sparse with respect to $f(n)$ consist almost entirely of zeros.

$M^{n}(R, S)$ is said to be a class of dual-sparse matrices with associated function $f(n)$ if $\left(n-r_{i}\right)<f(n)$ and $\left(n-s_{i}\right)<f(n), i=1(1) n$. We also consider a graph to be sparse or dual sparse if the incidence matrix of the graph is sparse or dual-sparse respectively.

2. Asymptotic enumerations. The first result which is solved by the technique for deriving asymptotics from the inclusion-exclusion formula is a slight generalization of the theorem of Erdös and Kaplansky [1], who showed that if $A \in M^{n}(n-k, n-k), k<(\log n)^{3 / 2-\epsilon}$, then for sufficiently large $n$

$$
\left|(\operatorname{Perm} A) e^{k} / n !-1\right|<n^{-c},
$$

where $c$ is a positive constant depending only on $\epsilon$. The technique used here is very close to that which was used in [1]. Note that Perm $\mathrm{A}$, the permanent of $A$, is the number of permutations which "lie in" the "frame" $A$, and thus this is a problem in permutations with restricted positions. We proceed to state our result:

Theorem 2.1. Let $A \in M^{n}(R, S), M^{n}(R, S)$ a class of dual-sparse matrices with $f(n)=(\log n)^{1-\epsilon}$. Then the number of zeros in $A$ is given by $n^{2}-\sum r_{i}=L$, and

$$
\text { Perm } A=n ! e^{-L / n}\left(1+o\left(n^{-1+\delta}\right)\right),
$$

where $\delta$ is arbitrarily small for $n$ sufficiently large. Hereafter, this qualification of order terms shall not be stated explicitly. It is assumed that $\delta$ and $\epsilon$ are arbitrarily small numbers when $n$ is sufficiently large.

This theorem, though broader than Erdös and Kaplansky's result, is less sharp. Indeed, Theorem 2.1 gives their result as a corollary but only with the stronger restriction $k<(\log n)^{1-\epsilon}$, and a weaker error 
bound. N. S. Mendelsohn [2] has derived a full asymptotic series for Perm $A$ under stronger restrictions but gives no error bounds.

A graph $G$ consists of a set of vertices $\{1,2, \cdots, n\}$ and a set of edges, which are ordered or unordered pairs of vertices $(i, j)$. If the edges are ordered pairs the graph is called directed; otherwise, it is undirected. We assume that all graphs are without loops, i.e. $(i, i)$ cannot be an edge, $i=1(1) n$. A complete graph contains all edges between vertices that may be formed. A hamiltonian circuit in a graph is an $n$-tuple of edges from the graph:

$$
\left(\left(i_{0}, i_{1}\right),\left(i_{1}, i_{2}\right), \cdots,\left(i_{n-1}, i_{n}\right)\right),
$$

where $i_{0}=i_{n}$ but otherwise no vertex is used twice: $i_{j} \neq i_{k}$ for $k \neq j, j, k$ $=1(1) n$. The number of hamiltonian circuits in a graph $G$ is denoted by \#h.c. $(G)$.

The following result is obtained using the inclusion-exclusion technique for asymptotics on graphs.

TheOREM 2.2. Let the directed graph $G$ on $n$ vertices be dual sparse, with $f(n)=(\log n)^{1-\epsilon}$. If $L$ directed edges may be added to $G$ to achieve a complete directed graph, then

$$
\text { \#h.c. }(G)=(n-1) ! e^{-L / n}\left(1+o\left(n^{-1+\delta}\right)\right) .
$$

In a directed graph, an edge $(i, j)$ is said to be incident from the vertex $i$ and incident to the vertex $j$. A corollary to Theorem 2.2 therefore is: if $G$ is a directed graph on $n$ vertices with the same number, $n-k-1$, edges incident to and from each vertex, $k<(\log n)^{1-\epsilon}$, then

$$
\text { \#h.c. }(G)=(n-1) ! e^{-k}\left(1+o\left(n^{-1+\delta}\right)\right) \text {. }
$$

We also may derive from Theorem 2.2:

CoROLlary. Let the nondirected graph $G$ on $n$ vertices be dual sparse with $f(n)=(\log n)^{1-e}$. If $L$ edges may be added to $G$ to achieve a complete graph, then

$$
\text { \#h.c. }(G)=1 / 2(n-1) ! e^{-2 L / n}\left(1+o\left(n^{-1+\delta}\right)\right) \text {. }
$$

The final result obtained using the inclusion-exclusion technique for asymptotics, deals with the number of matrices in the class $M^{n}(R, S), \# M^{n}(R, S)$. This problem was suggested by H. J. Ryser [3].

TheOREM 2.3. Let $M^{n}(R, S)$ be a collection of sparse matrices with $f(n)=(\log n)^{1 / 4-4}$. Then 


$$
\begin{aligned}
\# M^{n}(R, S)= & \frac{\left(\sum r_{i}\right) !}{\Pi r_{i} ! \Pi s_{i} !} \exp \left[\frac{-1}{2\left(\sum r_{i}\right)^{2}}\left(\sum r_{i}\left(r_{i}-1\right)\right)\left(\sum s_{i}\left(s_{i}-1\right)\right)\right] \\
& \cdot\left(1+o\left(n^{-1+\delta}\right)\right) .
\end{aligned}
$$

In the formula above, all sums and products range from 1 to $n$. Particularizing Theorem 2.3 to the evaluation of $\# M^{n}(k, k), k$ $<(\log n)^{1 / 4-\epsilon}$, we obtain

$$
\# M^{n}(k, k)=\frac{(n k) !}{k !^{2 n}} e^{-(k-1)^{2} / 2}\left(1+o\left(n^{-1+\delta}\right)\right) .
$$

3. Probabilistic asymptotic enumerations: first moments. Combining Theorem 2.3 with Theorem 2.1, it is not difficult to obtain the following "probabilistic" result:

Theorem 3.1. Assume $2 \leqq k<(\log n)^{1 / 4-e}$. Then

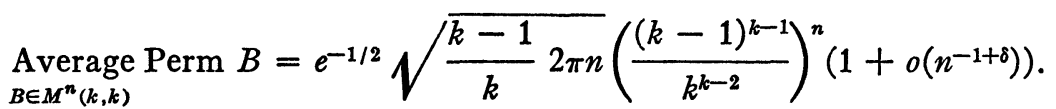

To derive a parallel theorem for the number of hamiltonian circuits in a sparse graph, we must first derive an intermediate result corresponding to Theorem 2.3. Using the correspondence between graphs and their incidence matrices, this is achieved by an argument involving equivalence classes in $M^{n}(k, k)$ followed by invoking Theorem 2.3 itself.

Let $D G_{n}(k, k)$ be the class of directed graphs on $n$ vertices with $k$ edges incident to and from each vertex.

LEMma. Let $D G_{n}(k, k)$ be a class of sparse graphs with $f(n)=(\log n)^{1 / 4-e}$. Then the cardinality of $D G_{n}(k, k)$,

$$
\# D G_{n}(k, k)=\frac{(n k) !}{(k !)^{2 n}} e^{-\left(k^{2}+1\right) / 2}\left(1+o\left(n^{-1+\delta}\right)\right) .
$$

This lemma, in combination with Theorem 2.2 gives

THEOREM 3.2. For $D G_{n}(k, k)$ a class of sparse graphs with $2 \leqq k$ $<(\log n)^{1 / 4-e}$,

$$
\begin{aligned}
\underset{G \in D G_{n}(k, k)}{\text { Average \#h.c. }(G)=} & e^{1 / 2} \sqrt{\frac{k-1}{k} 2 \pi\left(n^{-1}\right)}\left(\frac{(k-1)^{k-1}}{k^{k-2}}\right)^{n} \\
& \cdot\left(1+o\left(n^{-1+\delta}\right)\right) .
\end{aligned}
$$


4. Probabilistic asymptotic enumeration: the second moment. In this section, a great amount of exacting calculation is required to find the second moment of the permanent of a matrix $B$, as $B$ ranges over the class $M^{n}(k, k)$. The formula derived is contained in the following theorem.

THEOREM 4.1. Let $3 \leqq k<(\log n)^{1 / 4-e}$. Then

$$
\begin{aligned}
\underset{B \in \mathcal{M}^{n}(k, k)}{\text { Average }}(\operatorname{Perm} B)^{2}= & e^{-1}\left(\frac{k-1}{k}\right) 2 \pi n\left(\frac{(k-1)^{k-1}}{k^{k-2}}\right)^{2 n} \\
& \cdot\left(1+\frac{1}{4(k-1)^{4}}+O\left(\frac{1}{k^{6}}\right)\right)\left(1+O\left(n^{-1 / 2+\delta}\right)\right) .
\end{aligned}
$$

As a direct corollary of this and Theorem 3.1, we have the following "strong" probabilistic result.

COROLlary. Let $k=g(n)$ a monotonically increasing integer valued function of $n$, not bounded above, such that $g(n)<(\log n)^{1 / 4-\epsilon}$. Then ranging over all $n$, with $k=g(n)$, almost all matrices in $M^{n}(k, k)$ have permanent asymptotically equal to Average ${ }_{B \in M^{n}(k, k)}$ Perm $B$.

This is an immediate consequence of the fact that the second moment of Perm $B$ is equal to the square of the first moment asymptotically as $n$ and $k$ approach infinity.

5. Suggested further work. The author believes that the approach used to arrive at the estimates of $\$ 2$ of this work, i.e. the sieve formula, may be broadened by using the generalized inclusion-exclusion formula to arrive at asymptotics. A result which should be derivable from this technique is stated here as a conjecture.

ConjeCture 1. Let $A \in M^{n}(R, S)$, a sparse class of matrices with $f(n)=(\log n)^{1-\epsilon}$. Assume $r_{i}>0, s_{i}>0$ for all but $\mathrm{O}(\log n)$ integers $i=1(1) n$. Let $L$ be the total number of ones in $A$. Finally, Let $E(m)$ measure the number of permutation matrices having exactly $m$ ones in positions in common with ones of $A$. Then

$$
E(m) \sim\left(\begin{array}{l}
L \\
m
\end{array}\right)(n-m) ! e^{-(L-m) /(n-m)},
$$

asymptotically in $n$.

Note that in the special case $L=n, r_{i}=s_{i}=1$ for $i=1(1) n$, this reduces to the generalized Problème des récontres. The proof of this formula should be straightforward and is recommended as a research problem. It would be very useful in certain applications of combinatorics, such as finding higher moments in probabilistic asymptotics (see [4]). 
Another natural problem which suggests itself is to find the second moment of \#h.c. $(G)$ as $G$ varies over the class $D G_{n}(k, k)$. This would be the graph parallel of the matrix Theorem 4.1. One might hope for a strong result similar to the corollary to Theorem 4.1.

A third area of study would be to try to asymptotically enumerate the number of (properly limited) sparse matrices in a dual-sparse frame. Almost all the results of this work can be stated in this way, although they are trivial cases. Much of the work that went into proving Theorem 4.1, however, involved a specific nontrivial problem of this nature.

Finally, we offer two conjectures of surpassing difficulty, which are merely suggested by the results above. The author can suggest no line of attack for their solution.

Conjecture 2. We note that, as an extension of the work [1], Yamamoto showed [5] that for a dual sparse matrix $A \in M^{n}(n-k, n-k), k<n^{1 / 3-\epsilon}$, Perm $B \sim n ! e^{-k}$. Using Stirling's approximation and taking the $n$th root, we have

$$
(\text { Perm } B)^{1 / n} \sim(n-k) / e .
$$

At the same time, the corollary to Theorem 4.1 gives, in the $n$th root, that for $B \in M^{n}(k, k), k$ a function of $n$ increasing without hound, $k<(\log n)^{1 / 4-e}$, almost all matrices $B$ have

$$
(\operatorname{Perm} B)^{1 / n} \sim k / e .
$$

The conjecture is immediate that for $i$ intermediate between these two extreme ranges, asymptotically in $n$ for almost all $B \in M^{n}(i, i)$ we have

$$
(\operatorname{Perm} B)^{1 / n} \sim i / e .
$$

Conjecture 3. Erdös and Kaplansky pointed out that the permanent of an element of the class of matrices $M^{n}(n-k, n-k)$ counts the number of ways that a $(k+1)$ st row may be added to a specific latin rectangle of size $k \times n$. Leaning heavily on Conjecture 2, we may hope that in the $n$ squared root the number of latin squares, \#L.S., is asymptotic to the product of the average permanents of $B, B \in M^{n}(i, i)$, as $i$ goes from 1 to $n$. The conjecture implied is then

$$
\text { (\#L.S.) })^{1 / n^{2}} \sim n / e^{2} .
$$

\section{BIBLIOGRAPHY}

1. P. Erdös and I. Kaplansky, The asymptotic number of latin rectangles, Amer. J. Math. 68 (1946), 230-236. 
2. N. S. Mendelsohn, The asymptotic series for a certain class of permutation problems, Canad. J. Math. 8 (1956), 234-243.

3. H. J. Ryser, Combinatorial mathematics, Carus Math. Monograph, No. 14, 1963.

4. P. O'Neil, Asymptotics in random (0-1)-matrices, Preprint, February 8, 1969.

5. K. Yamamoto, On the asymptotic number of Latin rectangles, Japan. J. Math. 21 (1951), 113-119.

ibm Cambridge Scientific Center, 545 Technology Square, Cambridge, MASSACHUSETTS 02139 